УДК 624.012

\title{
ЗАДАЧА ОПТИМАЛЬНОГО ПРОЕКТИРОВАНИЯ ШПРЕНГЕЛЬНОЙ БАЛКИ ПРИ ДЕЙСТВИИ ПОСТОЯННОЙ НАГРУЗКИ
}

Кандидаты техн. наук Г.Л. Ватуля, Е.Ф. Орел, асп. С.Д. Синчук, С.А. Величко

\section{ЗАДАЧА ОПТИМАЛЬНОГО ПРОЕКТУВАННЯ ШПРЕНГЕЛЬНОЇ БАЛКИ ПРИ ДІЇ ПОСТІЙНОГО НАВАНТАЖЕННЯ}

Кандидати техн. наук Г.Л. Ватуля, Є.Ф Орел, асп. С.Д. Сінчук, С.А. Величко

\section{STRUCTURAL DESIGN OPTIMIZATION OF STRUT BEAM UNDER CONSTANT LOAD}

Candidates of techn. sciences G.L. Vatulia, PhD, E.F. Orel, postgraduate S.D. Sinchuk, S.A. Velichko

В статье рассматриваются вопросы поиска оптимального проекта статически определимой комбинированной конструкции в виде ипренгельной балки, загруженной постоянной нагрузкой. Предложена методика оптимизации конструкции с использованием метода Ньютона.

Ключевые слова: комбинированная система, целевая функция, уравнение оптимальности, частная производная, постоянная нагрузка, метод Ньютона.

У статі розглянуто питання пошуку оптимального проекту статично визначеної комбінованої конструкиї у вигляді шпренгельної балки, яка працює в умовах постійного навантаження. Запропоновано методику оптимізащії конструкиіі з використанням методу Ньютона.

Ключові слова: комбінована система, цільова функція, рівняння оптимальності, часткова похідна, метод Ньютона.

The authors describe the methodology of structural design optimization of composite structure using the Newton's method. The statically determinate strut beam was assumed in the article as a composite structure. The optimization was done under the constant loading. Structure's minimum weight, volume or cost was considered as a criterion of optimality. Brace location and strut beam rise are accepted as design variables. The authors assumed that efficiency function (volume function) is smooth and continuous and its minimum is located in the point where all partial derivative from function of the unknown variables are 
equal to zero. Utilizing of described procedure helps to provide the logical design of structure optimization taking into account loading condition, combined loading and system indeterminacy.

Keywords: composite structure, efficiency function, criterion of optimality, constant loading, partial derivative, Newton's method.

Введение. В настоящий момент большинство конструкций, которые эксплуатируются, исчерпали свой гарантийный проектный срок. Особенно это касается несущих конструкций транспортных сооружений. Современное состояние строительной отрасли позволяет проектировать и реализовывать прогрессивные расчетные схемы сооружений, в том числе комбинированных. Использование вариативных методов оптимального проектирования и компьютерного моделирования при проектировании пролетных строений мостов позволяет значительно улучшить показатели материалоемкости, стоимости и трудоемкости строительства и/или реконструкции.

Анализ основных публикаций. В данной работе, которая является продолжением исследований $[1,2]$, рассматриваются вопросы оптимизации статически определимой комбинированной конструкции, загруженной только постоянной нагрузкой. Разработано много различных методов решения разных по постановкам задач оптимального проектирования [3-5] и деления их по группам на непоисковые, где используются необходимые условия экстремума, и поисковые - методы математического программирования [6-8].

Целью исследований является составление алгоритма расчета оптимальной конструкции при проектировании шпренгельной балки пролетного строения пешеходного моста.

\section{Основной материал исследований.} Комбинированная конструкция является рациональной конструкцией по сравнению с балочной конструкцией за счет увеличения несущей способности. В качестве комбинированной конструкции нами рассматривается шпренгельная балка, конструкция которой представлена на рисунке.

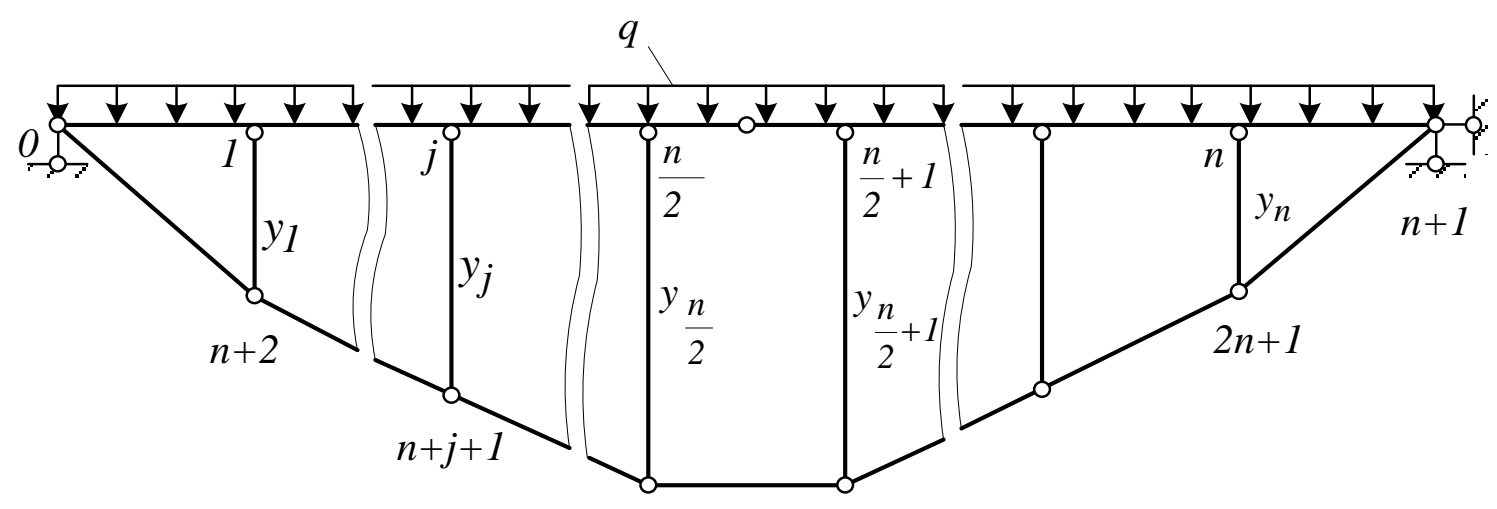

Рис. Схема шпренгельной балки

Для получения оптимального проекта шпренгельной балки необходимо найти такие значения переменных, чтобы затраты на изготовление балки (объем материала, масса и стоимость) были наименьшими. При этом должны удовлетворяться следующие условия: элементов

- условие прочности растянутых

$$
\sigma=\frac{N}{A} \leq \eta R_{y}
$$

- условие устойчивости сжатых стержней

$$
\sigma=\frac{N}{\varphi A} \leq \eta R_{y}
$$

- условие прочности для балки постоянного сечения, работающей на изгиб со сжатием

$$
\sigma=\frac{M}{W} \operatorname{sign} M+\frac{N}{A} \operatorname{sign} N \leq \eta R_{y},
$$


- конструктивные ограничения гибкости стержней $\lambda \leq \lambda$.

Раскроем последнее условие. Гибкость шарнирно опертого стержня

$$
\lambda=\frac{\ell}{i},
$$

где $l$ - длина стержня;

$$
i=\sqrt{\frac{I}{A}}=\eta_{1} \sqrt{A},
$$

где $\eta_{1}=\sqrt{\frac{I}{A^{2}}}$ - безразмерный коэффициент, зависящий от формы и размеров сечения.

Тогда

$$
\begin{gathered}
\lambda=\frac{\ell}{i}=\frac{\ell}{\eta_{1} \sqrt{A}} \\
V_{i, s}^{b}=\sup V_{i, j}^{b}=\frac{\ell}{\eta R_{y}}\left\{\frac { A } { W } \left[\frac{q \ell}{2} x_{i, s}-\frac{q x_{i, s}^{2}}{2}-\frac{q l^{2}}{8 y_{n / 2}}\left(y_{s-1}+\frac{y_{s}-y_{s-1}\left(x_{i j}-\right.}{z_{j}}\right.\right.\right. \\
\left.\left.\left.\left.-\sum_{k=1}^{s-1} z_{k}\right)\right)\right] \operatorname{sign}_{i, s}-\frac{q \ell^{2}}{8 y_{n / 2}} \operatorname{signN}\right\}
\end{gathered}
$$

элементы шпренгеля:

- стойки

$$
V_{j, n+j+1}=\frac{q l^{2} y_{j}}{8 y_{n / 2 \varphi_{j, n+j+1}} \eta R_{y}}\left(\frac{y_{j}-y_{j-1}}{z_{j}}-\frac{y_{j+1}-y_{j}}{z_{j+1}}\right), \quad j=1, \cdots, n / 2 ;
$$

- элементы нижнего пояса

$$
V_{j}=\frac{q l^{2}}{8 y_{n / 2} \eta R_{y}} \frac{\left(y_{j}-y_{j-1}\right)^{2}+z_{j}^{2}}{z_{j}}, \quad j=1, \cdots, n / 2
$$




$$
V_{n / 2+1}=\frac{q l^{2}}{8 y_{n / 2} \eta R_{y}}\left(\begin{array}{c}
n-2 \sum_{k=1}^{n / 2} z_{k}
\end{array}\right)
$$

Таким образом, уравнение для объема шпренгельной балки будет иметь следующий вид:

$$
V=V_{i, s}^{b}+2 \sum_{j=1}^{n / 2} V_{j, n+j+1}+2 \sum_{j=1}^{n / 2} V_{j}+V_{n / 2+1}
$$

Запишем уравнение оптимальности. Считая целевую функцию (функцию объема) непрерывной и гладкой, минимум ее будем отыскивать в точке, где все ее частные производные от функции по неизвестным переменным обращаются в ноль [9]. Таким образом, уравнение оптимальности будет представлять собой систему из $s=0,5 n$ нелинейных уравнений относительно всех топологических переменных:

$$
\frac{\partial V}{\partial y, z}=0
$$

При взятии производных от функции объема сжатых стержней необходимо получить выражения для вычисления производных от функций $\varphi(\lambda)$ по топологическим переменным:

$$
\begin{gathered}
\frac{\partial \varphi(\lambda)}{\partial y_{j}}=\left(3,333 \cdot 10^{-6} \lambda_{e f}^{2}-2,778 \cdot 10^{-4} \lambda_{e f}-2,5 \cdot 10^{-3}\right) \frac{\partial \lambda_{e f}}{\partial y_{j}} \\
\frac{\partial^{2} \varphi(\lambda)}{\partial y_{j}^{2}}=\left(\frac{\partial \lambda_{e f}}{\partial y_{j}}\right)^{2}\left(6,667 \cdot 10^{-6} \cdot \lambda_{e f}-2,778 \cdot 10^{-4}\right)
\end{gathered}
$$

Нужно отметить, что в общем случае уравнения оптимальности представляют собой систему нелинейных уравнений и аналитического решения не имеют. Решение находится методами последовательных приближений. Одним из таких методов является метод Ньютона, в котором система нелинейных уравнений заменяется решением системы линейных уравнений относительно приращений переменных при задании начальных произвольных значений.

Эти уравнения имеют следующий вид:

$$
\sum_{i=1}^{0,5 n} f_{j, i}^{\prime(m-1)} \Delta y_{i}^{(m)}=-f_{j}^{(m-1)}
$$

$$
\begin{gathered}
\text { где } f_{j}=\frac{\partial V}{\partial y_{j}}, \\
f_{j, i}^{\prime}=\frac{\partial f_{j}}{\partial y_{i}}, \\
j=1,2, \ldots, 0,5 n, \quad m=1,2, \ldots \quad-\quad \text { номер }
\end{gathered}
$$
приближения.

Свободные члены $f_{j}$ представляют собой невязки уравнений оптимальности при данных приближенных величинах переменных $y_{j}$. После решения системы определяются значения переменных следующего приближения:

$$
y_{j}^{(m)}=y_{j}^{(m-1)}+\Delta y_{j}^{(m)}
$$


Далее производится перерасчет шпренгельной балки и сравнение значений целевых функций двух приближений, полученных с учетом ограничений сечений элементов по гибкости. При

$$
V^{(m-1)}-V^{(m)} \approx 0
$$

процесс заканчивается.

Выводы. В результате расчета мы получаем методику поиска размеров оптимальной конструкции проектируемой шпренгельной балки. Следующим этапом работы является разработка алгоритмов для создания программы оптимизации комбинированных систем с учетом подвижной нагрузки, совместного загружения и статической неопределимости. Реализация всего комплекса мероприятий по оптимизации комбинированной системы позволит подойти вплотную к вопросу получения комбинированной сталежелезобетонной конструкции оптимального очертания.

\section{Список использованных источников}

1. Основы расчета и проектирования комбинированных и сталебетонных конструкций [Текст] / Э.Д. Чихладзе, Г.Л. Ватуля, Ю.П. Китов [и др.]; под ред. Э.Д. Чихладзе. - К.: Транспорт Украины, 2006. - $104 \mathrm{c}$.

2. Китов, Ю.П. Рационализация конструкций сталебетонных пролетных строений пешеходных мостов [Текст] / Ю.П. Китов, Г.Л. Ватуля // Наук.-техн. збірник. - К.: НДІБК, 2005. - Вип. 62, т. 2. C. $37-42$.

3. Виноградов, А.И. Проблема оптимального проектирования в строительной механике [Текст] / А.И. Виноградов. - Харьков: Вища шк., 1973. - 170 с.

4. Ройтман, М.И. Методы оптимального проектирования деформируемых тел [Текст] / М.И. Ройтман, Г.С. Шапиро. - М.: Наука, 1976. - 266 с.

5. Martin Philip Bendsoe Generating Optimal Topologies in Structural Design using a Homogenization Method [Текст] / Martin Philip Bendsoe, Noboru Kikuchi // Computer Methods in Applied Mechanics and Engineering. - North Holland. - 1998. - V. 71. - P. 197-224.

6. Клюев, С.В. Оптимальное проектирование строительных конструкций на основе эволюционных и генетических алгоритмов [Текст] / С.В. Клюев, А.В. Клюев. - Саарбрюкен: Lambert, 2011. - 128 с.

7. Чихладзе, Е.Д. Спрощена діаграма граничних станів сталебетонного елемента в задачах оптимізації стержневих конструкцій [Текст] / Е.Д. Чихладзе, М.Г. Черненко // Зб. наук. праць. - К.: НДІБК, 2003. - Вип. 59, кн. 1. - С. 310-317.

8. Ватуля, Г.Л. Визначення оптимальної області геометричних характеристик поперечного перерізу сталебетонної балки [Текст] / Г.Л. Ватуля, Е.А. Бєліков // Сб. науч. трудов. Днепропетровск: ПГАСА, 2011. - Вып. 61. - С. 74-78.

9. Китов, Ю.П. Некоторые соображения о критериях оптимальности [Текст] / Ю.П. Китов, Г.Л. Ватуля, М.А. Веревичева // Зб. наук. праць. - Харків: УкрДАЗТ, 2014. - Вип. 143. - С. 124-131.

$$
\text { Рецензент д-р техн наук, профессор А.А. Плугин }
$$

Ватуля Гліб Леонідович, канд. техн. наук, доцент, завідувач кафедри будівельної механіки та гидравліки Української державної академії залізничного транспорту. Тел.: (057) 730-10-70. E-mail: vatulya@kart.edu.ua. Орел Євген Федорович, канд. техн. наук, доцент кафедри колії та колійного господарства Української державної академії залізничного транспорту. Тел.: (057) 730-10-67.

Сінчук Софія Дмитрівна, аспирант кафедри будівельної механіки та гидравліки Украинської державної академії залізничного транспорту. Тел.: (057) 730-10-71.

Величко Сергій Анатолійович, магістр Украинської державної академії залізничного транспорту.

Vatulia Glib Leonidovich, cand. of techn. sciences, Associated Professor, Head of Building Mechanics and Hydraulics Department Ukrainian State Academy of Railway Transport. Tel.: (057) 730-10-70. E-mail: vatulya @ kart.edu.ua. Orel Yevhen Fedorovich, cand. of techn. sciences, Associated Professor of Track and Track Facilities Department Ukrainian State Academy of Railway Transport. Tel.: (057) 730-10-67.

Sinchuk Sofija Dmitrivna, Postgraduate of Building Mechanics and Hydraulics Department, Ukrainian State Academy of Railway Transport. Tel.: (057) 730-10-71.

Velichko Sergij Anatolijvich, Master of Science Ukrainian State Academy of Railway Transport. Tel.: (057) 730-10-71. 